\title{
Identification and Bioactive Potential of Endogenetic Fungi Isolated from Medicinal Caterpillar Fungus Ophiocordyceps sinensis from Tibetan Plateau
}

\author{
Meng-Yuan Guo, Yan Liu, Yan-Hua Gao, Tong Jin, Hong-Bo Zhang and Xuan-Wei Zhou* \\ Key Laboratory of Urban Agriculture (South) Ministry of Agriculture, and Engineering Research Center of Cell \& \\ Therapeutic Antibody, Ministry of Education, and School of Agriculture and Biology, Shanghai Jiao Tong University, \\ Shanghai 200240, People's Republic of China \\ *For correspondence: xuanweizhou@sjtu.edu.cn; xuanweizhou@163.com
}

\begin{abstract}
To characterize and explore the endogenetic fungi of Chinese Cordyceps from Tibetan plateau for their bioactive potential, more than 30 kinds of endogenetic fungi were routinely isolated from Chinese Cordyceps. Six selected isolates were further investigated on the mycelial growth rate, morphology and molecular characteristics and the content of nucleosides in the mycelial fermentation products. The results showed that all the six strains could grow well in the ordinary media. Analysis of their molecular and morphological characteristics confirmed that the six strains belonged to the genus of Fusarium, Mucor and Mortierella, respectively. The nucleosides including uridine, guanosine, adenosine and cordycepin had been detected in the mycelial fermentation products of all the 6 strains. The strain NS2-B1 was the best one in all strains, and could produce the most nucleosides with a yield of $1284.47 \pm 26.05 \mu \mathrm{g} / \mathrm{g}$. Meanwhile the strain NS2-B1 could produce more adenosine and cordycepin $(418.72 \pm 2.38 \mu \mathrm{g} / \mathrm{g}$ and $179.14 \pm 0.57 \mu \mathrm{g} / \mathrm{g})$, which was significantly higher than that of Chinese Cordyceps $(18.47 \pm 0.31 \mu \mathrm{g} / \mathrm{g}$ and $90.43 \pm 1.46 \mu \mathrm{g} / \mathrm{g} ; p<0.01)$. This study proved that the selected strain NS2-B1 had a potential application value. (C) 2017 Friends Science Publishers
\end{abstract}

Keywords: Ophiocordyceps sinensis; Endogenetic fungi; Identification; Metabolites

\section{Introduction}

Chinese Cordyceps is the rarest medicinal fungus of traditional Chinese medicine (TCM) grows in alpine grassland ecosystems on the Tibetan Plateau. It is a parasitic complex of a fungus (Ophiocordyceps sinensis) and a caterpillar, and a famous health care product used in China for more than 700 years (Wei et al., 2016). In the early classification system, Chinese Cordyceps was classified into the genus of Cordyceps sensu lato in Clavicipitaceae based on the classification method of morphology and anatomy. After molecular biological methods being used for modifying the classification of Cordycipitaceae and Clavicipitaceae, in 2007 some Cordyceps spp. were classified into a new family, Ophiocordycipitaceae (Sung et al., 2007). Wei et al. (2016) recently suggested that $O$. sinensis sexual state should entitle Chinese Cordyceps (Wei et al., 2016). In addition, Chinese Cordyceps holds a lot of bioactive components, such as nucleosides, Cordyceps polysaccharides, cordycepic acid, ergosterol and etc., which had a series of unique efficacy on inhibiting tumor growth, anticancer, control high blood sugar (Lo et al., 2006; Zhou et al., 2009a), significantly improving the immune function of the human body and other unique effects (Paterson, 2008; Zhang et al., 2009). However, its medicinal value, the effect of prevention and treatment disease, as well as the role played in the local economy, has been widely studied and recognized in the world (Zhang et al., 2009; Weckerle et al., 2010; Winkler, 2010).

The natural resources of Chinese Cordyceps growing in the special ecological environment are mainly distributed in the high latitude region of $3000-5000 \mathrm{~m}$ altitude in China, India, Nepal and Bhutan (Winkler, 2008; Zhang et al., 2010a; Zhou et al., 2014). In recent years, Chinese Cordyceps resources gradually decreased because of the frequent human activities, natural ecological environment deterioration, water shortage and other environmental factors, etc. In addition, with the prices of Chinese Cordyceps rising year by year, more and more people came to Cordyceps production area for collecting it, which brought a huge environmental pressure and ecological problems, and had attracted a lot of attentions from the government and scientific communities (Bian et al., 2008; Li et al., 2011). Modern research on basic biological theories has proved that organisms living in the same environment could produce genetic exchanges in the process of long term

To cite this paper: Guo, M.Y., Y. Liu, Y.H. Gao, T. Jin, H.B. Zhang and X.W. Zhou, 2017. Identification and bioactive potential of endogenetic fungi isolated from medicinal caterpillar fungus ophiocordyceps sinensis from tibetan plateau. Int. J. Agric. Biol., 19: 307-313 
evolution, while the organisms would produce similar metabolites (Stierle et al., 1993; Zhou et al., 2010). Therefore, endogenetic fungi of Chinese Cordyceps should also have the same or similar chemical constituents and pharmacological effects with its host (Chen et al., 2009). Based on this opinion, it increasingly became a hot research topic to isolate the endogenetic fungi from Chinese Cordyceps, confirm the fungus of the asexual generations (anamorph), and ferment these fungi by using artificial methods, etc. Early in 2003, Jiang and Yao reported that about 13 genera and more than 30 species that related to the anamorph of Chinese Cordyceps had been recorded. In accordance with the botanical nomenclature, some of them were only with genus name, but had not yet been identified to the level of species (Jiang and Yao, 2003). In 2010, Zhang et al. (2010b) reported that 572 strains of endogenetic fungi were isolated from natural Chinese Cordyceps. Most of the strains were classified to 37 different genera according to their growth and cultural-morphological characteristics (Zhang et al., 2010). Although a lot of endogenetic fungi were isolated from Chinese Cordyceps, a few of them were investigated on the secondary metabolite (Wei et al., 2016). In the present study, the colony morphological and microstructural characteristics coupled with the nucleotide sequences of the internal transcribed spacer (ITS) 1-5.8SITS2 genes of these endogenetic fungi were attempted to use for the confirmation of their classification status. High performance liquid chromatography (HPLC) was employed for analysis of nucleotides of the selected fungi. This study would provide a useful reference for identification of endogenetic fungi and laid the foundation for finding the substitute of Chinese Cordyceps.

\section{Materials and Methods}

\section{Fungal Materials, Media and Chemical Reagents}

Natural Chinese Cordyceps was collected from Ya'an Township, Baqên County, Nagqu Prefecture, Tibet, China. Cordycepes militaries fruit bodies were bought from Heilongjiang Xinyisheng Pharmaceutical Co., Ltd.

Three media were designed for using in this study. Potato dextrose agar (PDA) Yeast extract peptone sucrose (YPS) media were prepared according to the previous literature (Zhou et al., 2007). The fermentation medium consisted of dextrose $25 \mathrm{~g}$, maltose $15 \mathrm{~g}$, peptone $20 \mathrm{~g}$, $\mathrm{KH}_{2} \mathrm{PO}_{4} 0.8 \mathrm{~g}, \mathrm{MgSO}_{4} \cdot 7 \mathrm{H}_{2} \mathrm{O} 0.8 \mathrm{~g}$, water $1000 \mathrm{~mL}$, pH 5.0 , was used for the liquid-state fermentation of endogenetic fungi.

Standard substance of cordycepin, adenosine, guanosine and uridine purchased from Shanghai Aladdin Bio-Chem Technology Co., Ltd. Methanol (chromatographically pure) was purchased from Fisher Scientific Worldwide (Shanghai) Co., Ltd. Reagents used for preparation of the media are chemically pure, and the other reagents are analytically pure (Sinopharm Chemical
Reagent Co., Ltd, Shanghai, China).

\section{Isolation and Purification of Endogenetic Fungi}

Chinese Cordyceps samples including stroma and sclerotia were washed with sterilized water, and then were designed to be treated with $75 \%(\mathrm{v} / \mathrm{v})$ ethanol and then washed with sterilized water for three times. The sterilized stroma of Chinese Cordyceps was cut into pieces $(0.5 \times 0.5 \times 0.5 \mathrm{~cm})$, the sclerotia kept in original status and was cut into pieces of $0.5 \mathrm{~cm}$. The cutting materials were obliquely inserted into Petri dishes with PDA medium, and then the Petri dishes were placed at $20^{\circ} \mathrm{C} \sim 25^{\circ} \mathrm{C}$ in the dark. After several days, fungi were observed growing from the stroma and sclerotia fragments in the plates. Individual hyphal tips of the various fungi were removed from the agar plates, placed on new PDA medium, and incubated at $25^{\circ} \mathrm{C}$ for at least 7 days. Each isolate was checked for purity and transferred to another agar plate by the hyphal tip method (Zhou et al., 2009b).

\section{Investigation of Mycelial Growth Rate}

Mycelial growth rate (GR) of the fungal colonies was calculated according to the method described in the previous literature (Barseghyan et al., 2011) with some modifications. Fungal strains were planted on PDA media in Petri dishes and inoculated at $25^{\circ} \mathrm{C}$ in the dark. After culture for 2 days, the diameters of the colonies was measured every $24 \mathrm{~h}$ using a vernier calipers and the related data was recorded.

\section{Observation of Colony Morphological and Microstructural Characteristics}

After mycelia were cultured for a few days and the mycelial clones were full of the Petri dishes. The morphology of the fungal clones that include peculiarities of the advancing (marginal) zone, aerial mycelium, colony color, and possible changes in the reverse colony surface were observed and recorded.

Analysis of the spore-release mechanism the spore's developmental characteristics was based on the described methods in previous literature (Zhou et al., 2009b). The microscopic characteristics of the fungi were observed with an Olympus BX51TRF microscope (Olympus, Ina-shi, Nagano, Japan). Microscopic and macroscopic photos were taken with a photo camera (Canon Co., Ltd, Tokyo Japan). Mycelial fragments were mounted on a microscope slide and examined in water or potassium hydroxide solution (KOH $10 \%)$ using a light/dark field microscope with or without phase contrast at high magnifications up to $400 \times$.

\section{Analysis of ITS Sequences of Fungal Isolates}

Preparation of fungal mycelia and isolation of DNA from mycelia were according to the cetyltrimethyl ammonium bromide method as described by previous literature 
(Zhou et al., 2007). The quality and concentration of DNA samples were examined by agarose gel electrophoresis and spectrophotometer analysis.

Based on the conserved sequences of the nuclear ribosomal ITS1, 5.8 S rDNA, and ITS2 region of fungi, the primers ITSF (5'-TCCGTAGGTGAACCTGCGG-3') and ITSR (5'-TCCTCCGCTTATTGATATGC-3') were designed and synthesized (Sangon Biotech, Shanghai, China). The PCR amplification was performed in a PTC$100^{\mathrm{TM}}$ programmable (MJ Research, Inc., UK) for 35 cycles $\left(94^{\circ} \mathrm{C}\right.$ for $30 \mathrm{~s}, 55^{\circ} \mathrm{C}$ for $1 \mathrm{~min}$, and $72^{\circ} \mathrm{C}$ for $2 \mathrm{~min}$ ) followed by extension for $10 \mathrm{~min}$ at $72^{\circ} \mathrm{C}$. The PCR products were purified using the Gel Extraction Mini Kit (Watson BioTechnologies, Shanghai, China), ligated to pMD18-T vectors (TaKaRa Biotechnology, Dalian, China), transformed into $E$. coli strain DH5 $\alpha$, and then sequenced in the Sangon Biotech (Shanghai) Co., Ltd. (Shanghai, China).

Analysis of the rDNA ITS sequences were performed by the comparison of the sequences and the sequences in GenBank (http://www.ncbi.nlm.nih.gov/nuccore/) using the basic local alignment search tool (BLAST). DNA sequences were edited and aligned using the Clustal W multiple sequence alignment programs at the Biology Workbench Version 3.2 (Thompson et al., 1994). Final multiple alignments were visually checked and adjusted manually when necessary. Phylogenetic analysis was conducted by the nucleotide sequence of ITS1-5.8S-ITS2 region using the molecular evolutionary genetics analysis (MEGA) software version 2.1 (Tamura et al., 2011).

\section{Preparation of Fungal Mycelia and Extraction of Various Nucleosides}

At first, two agar blocks obtained from a stock culture tube were inoculated into a $250 \mathrm{~mL}$ Erlenmeyer flask with $50 \mathrm{~mL}$ YPS medium and then placed on a shaker at $210 \mathrm{rpm}$ at $28^{\circ} \mathrm{C}$ for 5 days. Then $25 \mathrm{~mL}$ of above cultures were transferred to four 1L Erlenmeyer flasks with $250 \mathrm{~mL}$ of the fermentation medium, respectively. The fermentation was performed in these flasks on a $210 \mathrm{rpm}$ rotary shaker at $28^{\circ} \mathrm{C}$ for a week. The mycelium and supernatant was separated from the broth with centrifugation at $4000 \mathrm{~g}$ under $4^{\circ} \mathrm{C}$ for $10 \mathrm{~min}$. Then the fresh mycelium was dried using vacuum freeze drying machine (Alpha 1-2LD Plus, Christ, Germany) for $15 \mathrm{~h}$. The dried mycelia and supernatants were stored in a refrigerator at $-20^{\circ} \mathrm{C}$.

Meanwhile, Cordyceps samples were used as controls for the analysis of various nucleosides including uridine, guanosine, adenosine and cordycepin. Various samples treated with the similar method, were prepared to extract nucleosides, respectively.

After Cordyceps materials samples were ground into powder together in liquid nitrogen, approximate $1.0 \mathrm{~g}$ of the Cordyceps samples were precisely weighed and added into $10 \mathrm{~mL}$ methanol water $(50 / 50, v / v)$ in a $50 \mathrm{~mL}$ centrifuge tube which was subsequently placed in a ultrasonic machine for extracting various nucleosides at a power of 300 watt for $10 \mathrm{~min}$. After the centrifugation, the sample extraction procedure was repeated twice. Supernatant obtained from the three times centrifugation was mixed and exactly measured of its volume. The sample was filtrated through a $0.45 \mu \mathrm{m}$ filter prior to HPLC analysis. The extraction of nucleosides from Cordyceps samples and mycelia of the isolated fungi was also carried out in a similar procedure.

\section{Determination of Various Nucleosides}

All HPLC analysis work was carried out on a Waters 2696 separation module (Waters, Milford, MA, USA ), which consists of a Waters 2996 photo-diode array detector, an auto injector, and a reverse phase column (YMC-Pack Pro C18/250×4.6 mml. D.S-5 $\mu \mathrm{m} .12 \mathrm{~nm}$ ). Various solvent of the standard substance was consecutively injected five times to draw calibration curves. The determination condition of the samples was set as follows: the mobile phase adopted in the analysis consists of water and methanols were in the ratio $85: 15(v / v)$. The separation was conducted in isocratic elution with a flow rate of $0.8 \mathrm{~mL} / \mathrm{min}$. The detection wavelength of photo-diode array was set at $260 \mathrm{~nm}$ and the column temperature was $35^{\circ} \mathrm{C}$. The injection volume was 10 $\mu \mathrm{L}$ (Huang et al., 2009).

\section{Data Analysis}

Calculations of mean, standard deviation (SD), and $p$ values were performed on triplicate experiments using SPSS 19.0 software (SPSS Inc, NY, USA). The Student $t$ test was used to calculate $p$ values for comparison. Significant statistics were set at a $p$ value $<0.05$.

\section{Results}

\section{GR of the Endogenetic Fungi}

Six endogenetic fungi were selected for the investigation of GR from more than thirty isolates, and assigned as NS1-B, NS2-B1, NS2-B2, NS2-B3, NS2-B4 and NS2-Z, respectively. The GR of the six endogenetic fungi was showed in Fig. 1.

Among of these six endogenetic fungi, the GR of the fungal strains NS1-B and NS2-B1 showed a trend of the slowly growing before they are quickly growing in 8th days. The fungal strains NS2-Z, NS2-B2 and NS2-B3 grew quickly before they became slow. The fungal strain NS2-B4 grew steadily and with a GR of about $10.24 \pm 1.40 \mathrm{~mm} / \mathrm{d}$ in 6 th days. In all, the fungal strain NS2-B1 was the best one and with a GR of about $32.69 \pm 6.45 \mathrm{~mm} / \mathrm{d}$, which was significantly faster than other endogenetic fungi $(p<0.05)$. Fungal clones of six strains were full of petri dishes within 8 days (Fig. 1). 


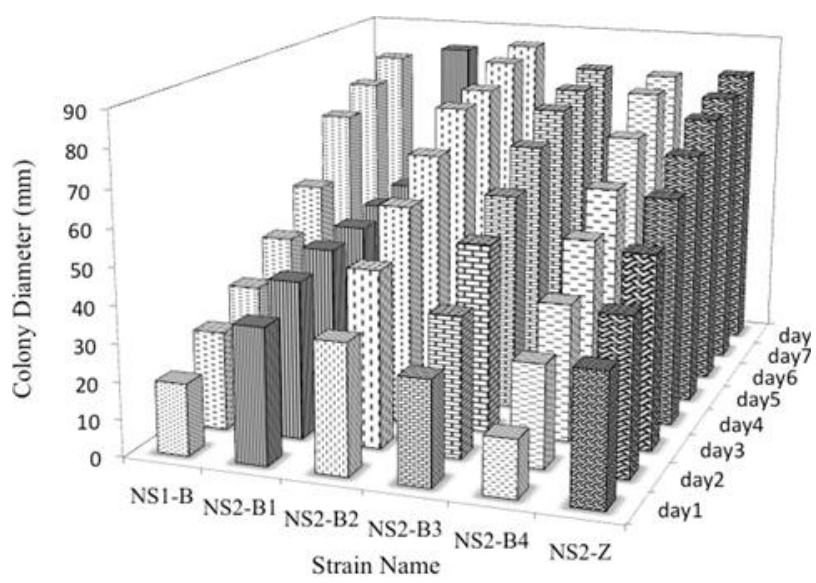

Fig. 1: GR of endogenetic fungi isolated from Chinese Cordyceps on PDA media $(n=3)$

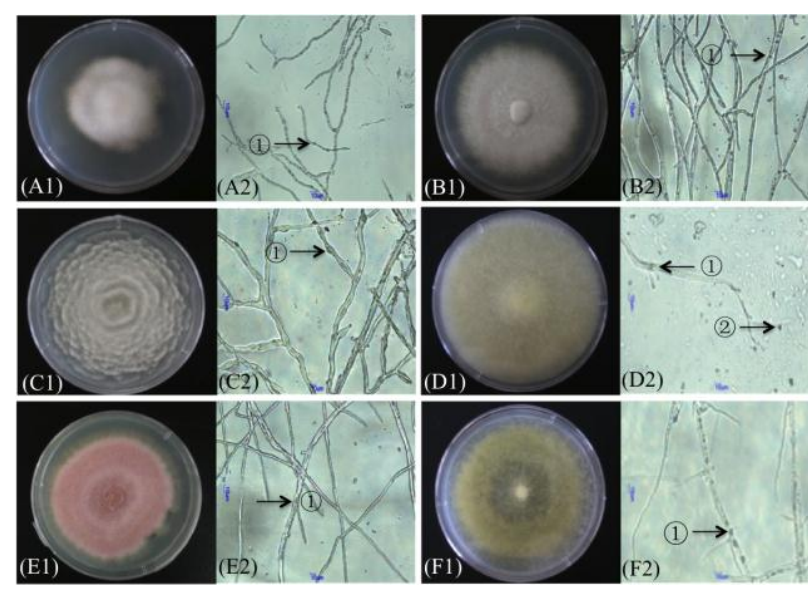

Fig. 2: Colony morphology and sporulation characteristics of endogenetic fungi isolated from Chinese Cordycpes $(400 \times)$. 1- morphological characteristic of mycelial colony on PDA; 2- microstructural characteristics of interlaced hyphae; (1)-chlamydospores; (2)-sporangiospore; A- fungal strain NS1-B; B- fungal strain NS2-B1; C- fungal strain NS2-B2; D- fungal strain NS2-B3; E- fungal strain NS2B4; F- fungal strain NS2-Z

\section{Morphological and Microstructural Characteristics of the Fungal Clones}

Colony morphological and microstructural characteristics of the six isolates were presented in Fig. 2. There were obvious differences on their colonial morphologies which were summarized in Table 1.

Microscope observation of mycelium culture showed that the strain NS1-B started to be white and villiform. It had aerial mycelia with short mycelia, 0-2 mediastinum, 27 diaphragms and branches (Fig. 2-a2). The strain NS2-B1 had thick and thin mycelia, diaphragm and branches. There were black chlamydospores in the mycelia (Fig. 2-b2).
The mycelia of the strain NS2-B2 was colorless and was white later. It has no diaphragms, but it had branches and light brown chlamydospores. Some mycelia top extended like break which was light brown (Fig. 2-c2). The strain NS2-B3 had two types of mycelium, thick and thin mycelium. The thin mycelia had colorless branches whose top can produce sporangiospore. The thick mycelia had light brown chlamydospores, branches and diaphragms. The strain NS2-B4 had thick and thin mycelia, with oval chalmydospores, diaphragms and branches. The strain NS2$\mathrm{Z}$ had thick and thin mycelia. The thick mycelia were light brown and had diaphragms and branches, with subglobose chlamydospores inside. The thin mycelia were colorless and had branches.

\section{Characteristics of Nuclear Ribosomal ITS1, 5.8 S rDNA, and ITS2 Sequences of the Endogenetic Fungi}

After the sequence alignment of the sequencing data and the sequences data in the GenBank, phylogenetic relationship analysis was performed using the MEGA software version 2.1 (Tamura et al., 2011). The phylogenetic tree was constructed from the specific nucleotide sequences of the ITS1-5.8S-ITS2 region of the six isolates. The results of phylogenetic tree analysis showed that all the strains were clustered into three groups, Fusarium, Mucor and Mortierella, using the ITS1-5.8S-ITS2 rDNA sequences (Fig. 3).

Among these isolates, the strain NS2-B4 ITS sequences showed $97 \%$ similarity with Fusarium acuminatum. The strain NS2-B1 ITS sequences showed 99\% similarity with $F$. torulosum. Coupled with the morphological characteristics above-mentioned, the strain NS2-B4 and NS2-B1 were preliminary classified into the genus of Fusarium. The strain NS2-Z ITS sequences showed $99 \%$ similarity with Mucor hiemalis. So it is most likely the genus of Mucor spp. The strain NS1-B ITS sequences showed $87 \%$ similarity with Mortierella alpine. So NS1-B was preliminary determined as the genus of Mortierella. The strain NS2-B2 and NS2-B3 ITS sequences showed $72 \%$ similarity with Mortierella gamsii. So the two fungi were most likely the genus of Mortierella.

\section{Analysis of Nucleosides Components from the Endogenetic Fungi}

The results of HPLC analysis showed that four kinds of nucleosides could be detected in Chinese Cordyceps, $C$. militaries fruit bodies and endogenetic fungi (Fig. 4).

Under the optimum chromatographic conditions, the retention time (Tr) of four kinds of standard reference substance including uridine, guanosine, adenosine and cordycepin, was $4.88,6.11,10.97$ and 13.86 , respectively. The detection wavelength of a chromatographic procedure was $260 \mathrm{~nm}$, the limit of detection was at the linear ranger of $0-200 \mu \mathrm{g} / \mathrm{mL}$. Based on the chromatographic conditions, 
Table 1: Colonial morphology and mycelial microstructure of six eodogenetic fungi

\begin{tabular}{|c|c|c|}
\hline Strains No. & Colony morphological characteristics & characteristics \\
\hline NS1-B & $\begin{array}{l}\text { Hard and dense white colony with radial } \\
\text { longitudinal grain }\end{array}$ & $\begin{array}{l}\text { Aerial mycelia with short mycelia, whose diameter was } 2.1-5.5 \mu \mathrm{m} \text {, light brown } \\
\text { chlamydospores inside, } 0-2 \text { mediastinum, 2-7 diaphragms and branches }\end{array}$ \\
\hline NS2-B1 & White, villiform and spread around & $\begin{array}{l}\text { thick and thin mycelia whose diameters were } 8.8-13.8 \mu \mathrm{m} \text { and } 1.7-2.7 \mu \mathrm{m} \text {, black } \\
\text { chlamydospores inside, diaphragm and branches. }\end{array}$ \\
\hline NS2-B2 & White and spread around like petal & $\begin{array}{l}\text { colorless and later white mycelia whose diameter was } 3.7-6.9 \mu \mathrm{m} \text {; no } \\
\text { diaphragms, but with branches and light brown chlamydospores; light brown } \\
\text { break extended from the top of some mycelia. }\end{array}$ \\
\hline NS2-B3 & Yellow, flocculent and spread around & $\begin{array}{l}\text { Thick mycelia whose diameter was } 8.8-16.7 \mu \mathrm{m} \text { with light brown } \\
\text { chlamydospores, branches and diaphragms; thin mycelia whose diameter was 1.2- } \\
1.7 \mu \mathrm{m} \text { with colorless branches whose top can produce sporangiospore. }\end{array}$ \\
\hline NS2-B4 & Red, villiform and spread around & $\begin{array}{l}\text { Thick and thin mycelia whose diameters were } 1.5-2.7 \mu \mathrm{m} \text { and } 8.0-14.5 \mu \mathrm{m} \text { with } \\
\text { oval chalmydospores, diaphragms and branches. }\end{array}$ \\
\hline NS2-Z & Yellow, flocculent and radial & $\begin{array}{l}\text { Thick, light brown mycelia whose diameter was } 8.6-23 \mu \mathrm{m} \text { with branches, } \\
\text { diaphragms and subglobose chlamydospores; thin colorless mycelia whose } \\
\text { diameter was } 1.0-2.0 \mu \mathrm{m} \text { with branches. }\end{array}$ \\
\hline
\end{tabular}

the standard curves of the four kinds of standard reference substances were established, the regression equations of calibration curves and their coefficients were consequently calculated as follows: for uridine, $\mathrm{Y}=18779 \mathrm{X}+48586$ $\left(\mathrm{R}^{2}=0.999\right)$; for guanosine, $\mathrm{Y}=16669 \mathrm{X}+24111\left(\mathrm{R}^{2}=0.999\right)$; for adenosine, $\mathrm{Y}=26705 \mathrm{X}+20259 \quad\left(\mathrm{R}^{2}=0.999\right)$; for cordycepin, $\mathrm{Y}=21244 \mathrm{X}+8651.3\left(\mathrm{R}^{2}=0.999\right)$. The separation was conducted in isocratic elution with a flow rate of 1.0 $\mathrm{mL} / \mathrm{min}$, the detection wavelength of the photo-diode array was set at $210-400 \mathrm{~nm}$, the column temperature was $35^{\circ} \mathrm{C}$ and the injection volume was $10 \mu \mathrm{L}$.

The yield of various nucleosides from different samples was summarized in Table 2. For the detection of total nucleoside, $C$. militaries fruit bodies held the highest yield of $13080.27 \pm 12.39 \mu \mathrm{g} / \mathrm{g}$. Among the isolated fungi, both the strains NS2-B1 and NS2-Z were with a higher capacity of the production of total nucleosides, and with the yields of $1284.47 \pm 26.05 \mu \mathrm{g} / \mathrm{g}$ and $842.17 \pm 1.30 \mu \mathrm{g} / \mathrm{g}$, which was significantly higher than other strains $(p<0.05)$. For the isolated strains, the strain NS2-B1 was the best one for the producing uridine, and with a yield of $521.27 \pm 19.66 \mu \mathrm{g} / \mathrm{g}$ that was significantly higher than other strains $(p<0.05)$. The strain NS2-Z was the best one for the production of guanosine, which was with a yield of $194.90 \pm 1.57 \mu \mathrm{g} / \mathrm{g}$, and was significantly higher than other strains $(p<0.05)$. The strains NS2-B1 could produce more adenosine and with a yield of $418.72 \pm 2.38 \mu \mathrm{g} / \mathrm{g}$, the strain NS2-Z could produce more cordycepin and with a yield of $164.44 \pm 0.15 \mu \mathrm{g} / \mathrm{g}$. In all, C. militaries fruit bodies contained a lot of various nucleosides, which was significantly higher that other materials. For the production of adenosine and cordycepin, the strain NS2-B1 and NS2-Z should have better capacities than Chinese Cordyceps.

\section{Discussion}

In recent years, more scientists would like to pay more attention to microorganisms from extreme environments in

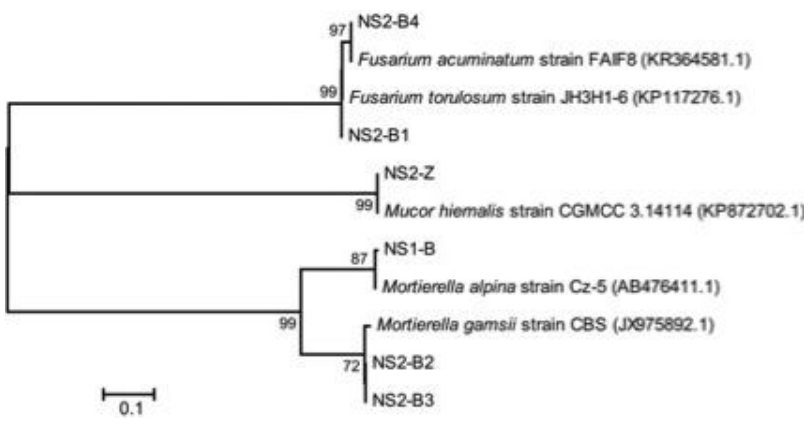

Fig. 3: Phylogenetic tree of isolated fungal strains. The phylogeny is constructed based on partial sequence of ITS1-5.8S-ITS2 region by MEGA 5.1 using Neighborjoining method with 1,000 bootstrap replicates. The scales indicate the number of nucleotide substitutions per site. GenBank accession IDs following the reference sequences are from NCBI

the field of life science research. For the research of Chinese Cordyceps, more and more investigators focused on their scientific interest in endogenetic fungi of Chinese Cordyceps and the fungal diversity (Zhang et al., 2010; Xia et al., 2015; Xia et al., 2016a; Xia et al., 2016b). Although endogenetic fungi of Chinese Cordyceps had been investigated and studied extensively, there was a little minor fault existing in the research process. For example, most of isolated endogenetic fungi were only classified based on their morphological descriptions.

Therefore, the establishment of the proper endogenetic fungi classification method combined with morphological and molecular characteristics is very necessary (Toledo $e t$ al., 2013). In the present study, more than thirty endogenetic fungi were routinely separated from natural Chinese Cordyceps. The colony morphological and microstructural characteristics coupled with the nucleotide sequences of ITS1-5.8S-ITS2 genes of these endogenetic fungi were 
Guo et al. / Int. J. Agric. Biol., Vol. 19, No. 2, 2017

Table 2: Regressive equation of different endogenetic fungi and Chinese Cordyceps

\begin{tabular}{llllll}
\hline Components samples & Uridine $(\mu \mathrm{g} / \mathrm{g})$ & Guanosine $(\mu \mathrm{g} / \mathrm{g})$ & Adenosine $(\mu \mathrm{g} / \mathrm{g})$ & Cordycepin $(\mu \mathrm{g} / \mathrm{g})$ & Nucleoside $(\mu \mathrm{g} / \mathrm{g})$ \\
\hline NS1-B & $182.95 \pm 1.50$ & $165.36 \pm 5.90$ & $18.45 \pm 0.81$ & $73.10 \pm 5.86$ & $439.86 \pm 9.25$ \\
NS2-B1 & $521.27 \pm 19.66$ & $165.34 \pm 4.58$ & $418.72 \pm 2.38$ & $179.14 \pm 0.57$ & $1284.47 \pm 26.05$ \\
NS2-B2 & $214.94 \pm 1.43$ & $113.15 \pm 11.15$ & $13.79 \pm 7.49$ & $42.33 \pm 1.07$ & $384.21 \pm 8.77$ \\
NS2-B3 & $247.38 \pm 5.65$ & $154.26 \pm 2.06$ & $215.88 \pm 0.45$ & $89.65 \pm 0.34$ & $707.17 \pm 3.62$ \\
NS2-B4 & $127.07 \pm 0.74$ & $56.02 \pm 2.41$ & $24.59 \pm 0.099$ & $13.67 \pm 0.08$ & $221.35 \pm 1.63$ \\
NS2-Z & $237.21 \pm 1.66$ & $194.90 \pm 1.57$ & $245.62 \pm 1.49$ & $164.44 \pm 0.15$ & $842.17 \pm 1.30$ \\
O. sinensis & $1392.73 \pm 1.84$ & $789.53 \pm 0.71$ & $18.47 \pm 0.31$ & $90.43 \pm 1.46$ & $2291.17 \pm 1.06$ \\
C. militaries & $4349.00 \pm 5.70$ & $1495.80 \pm 0.53$ & $2215.17 \pm 4.02$ & $5020.30 \pm 3.40$ & $13080.27 \pm 12.39$ \\
\hline
\end{tabular}

Notes: Bioactive component content was calculated in per gram dry mycelia or dry Cordyceps materials

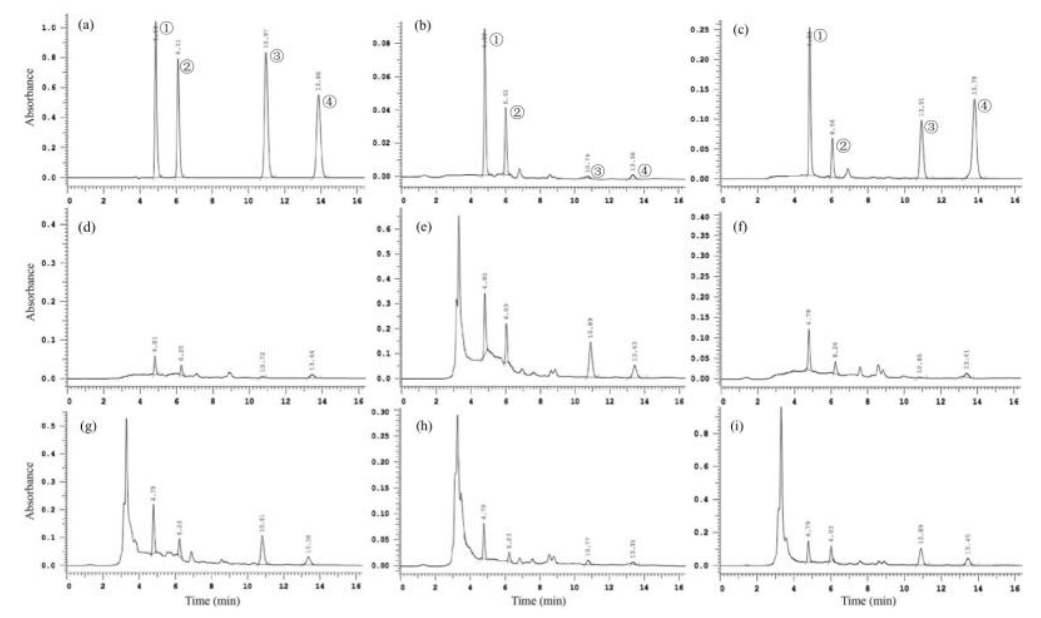

Fig. 4: HPLC analysis of four kinds of metabolites in various samples. (a): HPLC chromatograms of four kinds of metabolites; (b): for Chinese Cordyceps; (c): for Cordycepes militaries; (d): for fungal strain NS1-B; (e): for fungal strain NS2-B1; (f): for fungal strain NS2-B2; (g): for fungal strain NS2-B3; (h): for fungal strain NS2-B4; (i): for fungal strain NS2-Z. (1) Uridine (2) Guanosine (3) Adenosine (4) Cordycepin

attempted to use for the confirmation of their classification status. The results showed that six strains belonged to Fusarium spp., Mucor spp. and Mortierella spp., respectively, which should belong to the genus of mycoflora from Chinese Cordyceps that inhabit the area. For example, Zhang et al. (2010) reported that Fusarium spp. was the predominant fungi in grass grassland. Mucor spp. and Mortierella spp. were the common genus in 4 types of grassland, polygonum viviparum, grass steppe, swamp meadow and kobresia meadow (Zhang et al., 2010). After analyzing the types of grassland in Chinese Cordyceps habitats, the results of fungal classification in this study should therefore be reliable, and accordance with the previous reports (Zhang et al., 2010). However, little of literatures referred to morphological and molecular characteristics of these fungi. The detailed descriptions related to their morphological and molecular characteristics were presented in this study, which could improve and perfect the isolation and identification of endogenetic fungi in study of Chinese Cordyceps, while it will lay the foundation for further exploitation and utilization of those strains.
The organisms that lived in the same environment for a long time perhaps held similar metabolites. For example, taxol (a product using for treatment of breast cancer) was extracted from the bark of yew early, the fungi isolated from the bark of yew were lately found to produce taxol of the same chemical constitution (Zhou et al., 2010). In recent years, some fungi isolated from Chinese Cordyceps had been found to have the same or similar components and pharmacological functions with their host's materials (Jin et al., 2006; Zhou et al., 2014). Two theories, gene transfer theory (Shaw, 1993) and endosymbiotic theory (Liang, 1999), were employed for the explanation of these complicated natural phenomena. Based on these theories, most of researchers paid attention to the anamorph of Chinese Cordyceps, and its metabolites, such as cordycepin, cordycepic acid and other nucleosides, etc., but ignored the active constituent from other endogenetic fungi. Six fungal strains used in this experiment weren't anamorph of Chinese Cordyceps. However, HPLC analysis confirmed that the six strains could produce various nucleosides similar to the active components from Chinese Cordyceps, which was the accordance with a recent publication (Ai et al., 2016). 
Furthermore, two selected strains, both NS2-B1 and NS2-Z, were with the higher capacities for producing the adenosine and cordycepin that was a representative component in natural Chinese Cordyceps recorded in Chinese pharmacopoeia. NS2-B1 was with a potential capacity for the production of adenosine of $418.7 \pm 2.378 \mu \mathrm{g} / \mathrm{g}$, the cordycepin of $179.1 \pm 0.573 \mu \mathrm{g} / \mathrm{g}$. NS2-Z could produce the adenosine of $245.6 \pm 1.488 \mu \mathrm{g} / \mathrm{g}$ and the cordycepin of $164.4 \pm 0.145 \mu \mathrm{g} / \mathrm{g}$. This study provided quality resources for further using endogenetic fungi for the production of cordycepic acid and cordycepin.

\section{Acknowledgements}

The author would like to acknowledge Director Buyong Jiacuo and Xin Wang for their help to collect the natural Chinese Cordyceps samples. This work was financially supported by Tibet Shenglong Industry Co., Ltd (No: 2013310031001210).

\section{References}

Ai, Z., Z.M. Qian, W.J. Li, F.Q. Yang, S.L. Chen and E.W. Li, 2016. Recent advances in the analysis of nucleosides in Chinese cordyceps. Mycosystema, 35: 388-403

Barseghyan, G.S., J.C. Holliday, T.C. Price, L.M. Madison and S.P. Wasser, 2011. Growth and cultural-morphological characteristics of vegetative mycelia of medicinal caterpillar fungus Ophiocordyceps sinensis G.H. Sung et al. (Ascomycetes) isolates from Tibetan plateau (P.R. China). Int. J. Med. Mushrooms., 13: 565-581

Bian, D., C. Li and X.H. Yang, 2008. Analysis of the situation of Grassland Degradation and it's mechanism of the alpine pastoral area in northwestern Tibet. J. Nat. Resour., 23: 254-262

Chen, Y., H. Guo, Z. Du, X.Z. Liu, Y. Che and X. Ye, 2009. Ecology-based screen identifies new metabolites from a Cordyceps-colonizing fungus as cancer cell proliferation inhibitors and apoptosis inducers. Cell. Prolif., 42: 838-847

Huang, L., Q. Li, Y. Chen, X. Wang and X. Zhou, 2009. Determination and analysis of cordycepin and adenosine in the products of Cordyceps spp. Afr. J. Microbiol. Res., 3: 957-961

Jiang, Y. and Y.J. Yao, 2003. Anamorphic fungi relatedto Cordyceps sinensis. Mycosystema, 22: 161-176

Jin, C., X.M. Wu and G.Y. Chen, 2006. Clinical application of Jinshuibao. Capital. Med., 12: 42-43

Li, W.C., J.J. Qiu and F. Qiu, 2011. Research of sustainable utilization of Cordyceps sinensis resources in Naqu region of Xizang Zizhiqu. Ecological Economy, 6: 112-114

Liang, Q.Z., 1999. The diversity of fungal secondary metabolites and their potential applications. Biodiv. Sci., 7: 145-150

Lo, H., T. Hsu, S. Tu and K. Lin, 2006. Anti-hyperglycemic activity of natural and fermented Cordyceps sinensis in rats with diabetes induced by nicotinamide and streptozotocin. Am. J. Chin. Med., 34: $819-832$

Paterson, R.R.M., 2008. Cordyceps-A traditional Chinese medicine and another fungal therapeutic biofactory? Phytochemistry, 69: 14691495

Shaw, M.W., 1993. The biodiversity of microorganisms and invertebrates: its role in sustainable agriculture. Exp. Arg., 29: 133

Stierle, A., G. Strobel and D. Stierle, 1993. Taxol and taxane production by Taxomyces andreanae, an endophytic fungus of Pacific yew. Science, 260: $214-216$
Sung, G.H., N.L. Hywel-Jones, J.M. Sung, J.J. Luangsa-Ard, B. Shrestha and J.W. Spatafora, 2007. Phylogenetic classification of Cordyceps and the clavicipitaceous fungi. Stud. Mycol., 57: 5-59

Tamura, K., D. Peterson, N. Peterson, G. Stecher, M. Nei and S. Kumar, 2011. MEGA5: molecular evolutionary genetics analysis using maximum likelihood, evolutionary distance, and maximum parsimony methods. Mol. Biol. Evol., 28: 2731-2739

Thompson, J.D., D.G. Higgins and T.J. Gibson, 1994. CLUSTAL W: improving the sensitivity of progressive multiple sequence alignment through sequence weighting, position-specific gap penalties and weight matrix choice. Nucleic. Acid. Res., 22: 4673-4680

Toledo, A.V., M.E. Simurro and P.A. Balatti, 2013. Morphological and molecular characterization of a fungus, Hirsutella sp., isolated from planthoppers and psocids in Argentina. J. Insect. Sci., 13: 59-64

Weckerle, C.S., Y. Yang, F.K. Huber and Q. Li, 2010. People, money, and protected areas: the collection of the caterpillar mushroom Ophiocordyceps sinensis in the Baima Xueshan Nature Reserve, Southwest China. Biodivers. Conserv., 19: 2685-2698

Wei, J.C., X.L. Wei, W.F. Zheng, W. Guo and R.D. Liu, 2016. Species identification and component detection of Ophiocordyceps sinensis cultivated by modern industry. Mycosystema, 35: 404-410

Winkler, D., 2010. Cordyceps sinensis: A precious parasitic fungus infecting Tibet. Field. Mycol., 11: 60-67

Winkler, D., 2008. Caterpillar Fungus (Ophiocordyceps sinensis) Production and sustainability on the Tibetan plateau and in the Himalayas. Asian. Medicine., 5: 291-316

Xia, F., Y. Liu, G.R. Shen, L.X. Guo and X.W. Zhou, 2015. Investigation and analysis of microbiological communities in natural Ophiocordyceps sinensis. Can. J. Microbiol., 61: 104-111

Xia, F., X. Chen, M.Y. Guo, X.H. Bai, Y. Liu, G.R. Shen, Y.L. Li, J. Lin and X.W. Zhou, 2016a. High-throughput sequencing-based analysis of endogenetic fungal communities inhabiting the Chinese Cordyceps reveals unexpectedly high fungal diversity. Sci. Rep., 6: 33437

Xia, F., Y. Liu, M.Y. Guo, G.R. Shen, J. Lin and X.W. Zhou, 2016b. Pyrosequencing analysis revealed complex endogenetic microorganism community from natural DongChong XiaCao and its microhabitat. BMC. Microbiol., 6: 196

Zhang, Y., L. Xu, S. Zhang, X. Liu, Z. An, M. Wang and Y. Guo, 2009. Genetic diversity of Ophiocordyceps sinensis, a medicinal fungus endemic to the Tibetan Plateau: Implications for its evolution and conservation. BMC. Evol. Biol., 9: 1-12

Zhang, Y.J., B.D. Sun, Sh. Zhang, M. Wang, X.Z. Liu and W.F. Gong, 2010a. Mycobiotal investigation of natural Ophiocordyceps sinensis based on culture-dependent investigation. Mycosystema, 29: 518527

Zhang, J.Z., X.R. Chen, C.D. Yang and L. Xue, 2010b. A study on the diversity of soil cultured fungi in the alpine grassland of Eastern Qilian Mountains. Acta. Pratacult. Sin., 19: 124-132

Zhou, X.W., L.J. Li and E.W. Tian, 2014. Advances in research of the artificial cultivation of Ophiocordyceps sinesis in China. Crit. Rev. Biotechnol., 34: 233-243

Zhou, X.W., Z.H. Gong, Y. Su, J. Lin and K.X. Tang, 2009a. Cordyceps fungi: natural products, pharmacological functions and developmental products. J. Pharm. Pharmacol., 61: 279-291

Zhou, X., H. Zhu, L. Liu, J. Lin and K. Tang, 2010. A review: recent advances and future prospects of taxol-producing endophytic fungi. Appl. Microbiol. Biotechnol., 86: 1707-1717

Zhou, X., Q. Li, J. Zhao, K. Tang, J. Lin and Y. Yin, 2007. Comparison of rapid DNA extraction methods applied to PCR identification of medicinal mushroom Ganoderma spp. Prep. Biochem. Biotechnol., 37: $369-380$

Zhou, X.W., W.L. Zheng, H.F. Zhu and K.X. Tang, 2009b. Identification of a taxol-producing endophytic fungus EFY-36. Afr. J. Biotechnol., 8: $2623-2625$

(Received 05 October 2016; Accepted 16 January 2017) 